Revista Iberoamericana. Vol. LXV, Núm. 187, Abril-Junio 1999; 305-325

\title{
MANUEL PUIG: DEL DELITO DE LA ESCRITURA AL ERROR GAY*
}

\author{
POR \\ JULIA ROMERO \\ Universidad Nacional de La Plata
}

El Delito de LA ESCRITURA ${ }^{1}$

La consideración del delito nos remite necesariamente a la legalidad y, consecuentemente, a la cuestión de lo nacional, a una zona sagrada en la que se incluyen y se excluyen modelos de lenguaje, de subjetividad, de relaciones institucionales. Hablar de nacionalismo en Argentina constituye en sí una paradoja ya señalada por la literatura y por la crítica. ${ }^{2}$ Escribir una literatura "propia" en una nación que admiraba modelos extranjeros fundó además de un problema más o menos presente a lo largo de distintas épocas, también una tradición de contradicciones que resulta su condición de posibilidad. Las funciones homogeneizantes que se destinaron a la literatura para inventar, mantener y reproducir la comunidad imaginada propiciaron el establecimiento de normas que regularon y fijaron una forma de escritura y un imaginario deseable y enseñable de identidades nacionales, relacionado siempre con valores ascendentes de la clase burguesa: la patria, la virilidad, la femineidad, la familia, la heterosexualidad, el orden, la salud, la higiene, y la consecuente

\footnotetext{
*El presente artículo surge en el contexto de una investigación realizada por el grupo que integro junto a Graciela Goldchluk, dirigido por José Amícola, de la Universidad Nacional de La Plata. El grupo se dedica al estudio de versiones éditas e inéditas de los trabajos originales del escritor argentino Manuel Puig, en posesión de sus herederos. A partir de 1996 el trabajo se centra en una edición crítico geneticista de El beso de la mujer araña para la colección Archivos de la UNESCO, que contiene el material previo a la redacción de la novela como las dos versiones, en manuscrito y en dactiloscrito, a las que aquí me refiero. El trabajo es una profundización de líneas de investigación que he abordado en trabajos anteriores y que aquí abordo en particular. Otra versión del trabajo, más suscinta, fue presentada al Congreso de Literatura Argentina organizado por la Universidad Nacional de Córdoba en octubre de 1997. Con relación a El beso de la mujer araña, un estudio de la forma cifrada de referir la historia argentina fue presentada en los Cuadernos Angers-La Plata; un adelanto del estudio de los pre-textos referidos a los filmes integra el volumen de Hispamérica (agosto de 1998).

1 Josefina Ludmer utiliza el tópico del delito para analizar las representaciones de "mujeres que matan"en la literatura argentina. Resulta sumamente productivo para analizar el corpus de la literatura, que permite arbitrar, reagrupar, repensar los textos. En este caso, me aparto de su modelo de análisis pero debo a la lectura de su ensayo la presente consideración del delito como la "escritura prohibida", con relación al campo intelectual.

${ }^{2}$ Borges en el ensayo "El escritor argentino y la tradición"; Sarlo (167).
} 
exclusión de todo aquello que no se aviniera a la construcción de esa metafísica política. Simultáneamente se tejió una red de escritura "en delito", una escritura prohibida que socavaba el paradigma desde su exclusión. Observar esos movimientos conduce a revelar que los debates culturales acerca de la cuestión nacional emergieron y se sucedieron en Argentina en coyunturas en que esas políticas homogeneizadoras se sintieron amenazadas. En el siglo XX, el primero de esos momentos ocurre alrededor de 1910, con las reflexiones que suscitó el Centenario de la Independencia; y el último, el período posperonista de 1955 hasta la década del 70, pero es a partir de 1974 cuando se produce una intensificación de los mecanismos de control ejercidos desde el Estado. Que ese proceso se haya dado en el marco de una ascendente violencia, desarrollado invariablemente con la participación de las Fuerzas Armadas, corrobora la idea de que los nacionalismos en Argentina se originaron como respuesta de ideologías amenazadas por la presencia de sectores sociales subalternos que se ofrecían como remanente a esa totalidad: a principios de siglo, el aluvión inmigratorio había puesto en escena una heterogeneidad de identidades y culturas que, en palabras de Eduardo Mallea fueron debilitando "la forma espiritual de nuestro pueblo", y adulterando un "producto natural". Respecto del proceso inmigratorio es clara su postura como uno de los intelectuales orgánicos que intervinieron posteriormente, pero que sintetiza la posición de los nacionalistas de la primera hora: "Me pareció que siendo un pueblo de origen sano, auténtico, rápido y pródigo de inteligencia, fundamentalmente desprendido [...] estábamos encaminándonos, por cierta complacencia en un vicio prematuro, a quién sabe qué nieblas, quién sabe qué destinos, quién sabe qué extravíos." Y más adelante: "Pero ante todo, era menester definir el origen del mal visible en la superficie del país. [...] Era un delito de la conciencia. El delito de esos hombres que habían suprimido sus propias raíces..."(66-67) Este discurso debe entenderse en el marco de la crisis de consenso y de poder resultante de la quiebra del sistema político oligárquico en Argentina de 1940. La emergencia de nuevos grupos -en general, hijos de aquellos inmigrantes - que resultaron del proceso de industrialización dieron lugar a una clase obrera en formación y junto a la transformación de las sociedades arcaicas significaron un nuevo peligro para aquella mentalidad. En la medida en que la oposición ideológica y de intereses crecian, también crecieron los mecanismos de coerción política y social y sus grados de elaboración.

Desde el primer gobierno de facto en la Argentina moderna encabezado por Uriburu en 1930, los gobiernos electorales tuvieron un ritmo de duración cada vez menor mientras que el de los gobiernos de facto se acrecentaba tanto los gobiernos conservadores destinados a restaurar el orden oligárquico como los populistas destinados a reestructurar el poder afirma Avellaneda - se acompañaron invariablemente con la intervención de las fuerzas armadas. A partir de 1960 tienen lugar las dos más extensas intervenciones: la del gobierno de la "Revolución Argentina" (1966-1973) y el gobierno del "Proceso de Reorganización Nacional" (1976-1983). La vida cultural argentina, entonces, se vio periódicamente condicionada por las formas del control político que van de la simple exclusión del horizonte literario de formas discursivas que no se condecían con el canon vigente (a principios de siglo) a la más inaudita persecusión y desaparición de personas que ha testimoniado la escena social y política. Por esa coyuntura, la década del setenta en Argentina fue también la escritura de las páginas más infames de la historia, matriz de un llanto retenido y de un 
duelo interminable. Escribir era, en muchos casos, sobreescribir la historia de desapariciones y del abuso del Estado, pero también convertirse en posible protagonista de una historia policial. Las víctimas de esa historia - se sabe - formaron un espectro que abarcaba desde los militantes de distintos partidos que deseaban luchar por los ideales de una sociedad más justa, a aquellos ciudadanos que pronunciaban ciertos nombres, leían ciertos autores, publicaban un libro, nombraban "malas palabras" o que figuraban ocasionalmente en una libreta telefónica. Tan absurda como la clasificación del idioma analítico del "Wilkins" de Borges, estos eran algunos de los criterios que regulaban la vida social de aquella época, en la que reinaba el mandato "el silencio es salud" y todo podía "desaparecer en el aire", según aseguraba una de las canciones del rock nacional, en una cita que seguramente no refería a la obra de Marshall Berman pero que no eludía la ironía que se entablaba con la de Marx. ${ }^{3}$ El espectro era amplio, los protagonistas muchos. Pero el esquema era fijo: la ideología de derecha era el dogma al que debía subordinarse la "Reorganización Nacional" ante el que cualquier indicio de contravención se aplicaban los procedimientos cada vezmás "uniformes". El delito era más que nunca el concepto que invadía la vida cotidiana y que ceñía de forma progresiva todas las libertades. En su nombre se paralizaban todas las formas del pensamiento y producción cultural, social, política, humana. En ese contexto, publicar también era una empresa de riesgo, ${ }^{4}$ narrar una historia convertía en muchas otras ocasiones a los escritores, al libro y a los editores en víctimas de la funesta historia de censura, y en una nueva forma de paralizar la resistencia. Estos casos de escritura ilegal, ilegítima por estar fuera de la ley del buen decir (cuyas normas habían sido arbitradas por los dictadores en función de una limpieza ideológica), concluyen en la fractura del campo intelectual, que tuvo consecuencias diversificadas, ${ }^{5}$ objeto de reflexiones y debates durante el período posterior, la reapertura democrática, que no estuvo exenta de acusaciones resentidas y de sentimientos culposos. ${ }^{6}$ En ese mismo contexto de debates es que Manuel Puig publica, en 1984, una declaración

\footnotetext{
${ }^{3}$ La letra de la canción corresponde al repertorio de Charly Gracía. Fragmento : "Los amigos del barrio pueden desaparecer,/ los cantores de radio pueden desaparecer, los que están en los diarios pueden desaparecer, la persona que amas puede desaparecer. / Los que están en el aire pueden desaparecer en el aire,/ los que están en la calle pueden desaparecer en la calle". La canción surgió en la etapa final de la dictadura, en 1982.

${ }^{4}$ El libro de Andrés Avellaneda guarda los testimonios de esa historia cultural que había sido censurada.

5 "Derrota" es el término que he encontrado en diversos estudios y declaraciones: derrota de la revolución, derrota de las actividades de resistencia, derrota como término implícito en su opuesto "Victoria". Entiendo que la denominación debería matizarse de alguna forma, circunscribirla a aquella coyuntura para no caer en generalidades y la consecuente desvalorización de las actividades de resistencia, que de todos modos permanecieron. De hecho, vivir a pesar de las condiciones que había impuesto la dictadura era, tanto para los que se quedaron como para los que tuvieron que exiliarse, resistir. De otro modo sólo se explicaría la caída del Régimen en el año 83 desde el punto de vista macrohistórico.

'José Luis de Diego hace referencia a estos debates frente a los cuales destaca la afirmación de Beatriz Sarlo, que recoloca una polémica que aparecía desplazada hacia el eje de oposición fuera/dentro de las fronteras del país y no como variaciones de la misma coyuntura: "la fractura del campo intelectual que el exilio significaba había sido el resultado de una operación victoriosa de la dictadura, y no de elecciones sólo recogidas por la libre voluntad de los sujetos" (223).
} 
sobre su relación con la censura, que toma por momentos los tonos de un manifiesto. Puig, exiliado en el sentido político, expatriado en el sentido cultural, escribe un texto donde lo que se muestra y se oculta no solo ilumina el posicionamiento del escritor respecto de la censura, la política peronista, y la codificación de la sexualidad, sino que devela y revela cómo su literatura había elaborado una respuesta cultural a esas instancias de la historia que trascienden lo personal, presentando una reflexión sobre las identidades que cuenta, desde una estética de lo intrascendente, la historia argentina.

II- Francine Masiello (13-14) afirma que cuando el Estado se encuentra en transición de una forma de gobierno a otra, de un período tradicionalista a un programa más modernizante, surge una alteración en la representación del género y puede encontrarse una configuración diferente de hombres y mujeres, modificada según el período histórico y la naturaleza de la crisis nacional. Cuando esas representaciones, agrego, sobrevienen en un período en el que la modernización parece retrasarse, retraerse a modalidades neomedievales, sobrevienen los mecanismos de censura frente a los hechos de la vida que continuaban la vía de modernización. El primer movimiento es el que habían tomado las representaciones en la literatura en las agitadas décadas de los 60 y 70 en Argentina, y es claro si se tiene en cuenta la inserción de las obras de Manuel Puig en el campo intelectual. Desde La traición de Rita Hayworth, de 1968, hasta Sangre de amor correspondido, de $1982,{ }^{7}$ puede leerse una historia en las representaciones del género en contrapunto con la política nacional, y también la causa en la que recaería la persecusión ideológica que se desplegaba con la fascistización progresiva del Estado. Esta circunstancia que Puig enumera en "Loss of Readership" sin embargo deja su huella en las novelas. Con El beso de la mujer araña inaugura una "trilogía del exilio", junto a Pubis angelical y Maldición eterna a quien lea estas páginas, novelas con las que el escritor se dedicará a realizar especialmente un ajuste de cuentas con la patria. Con El beso de la mujer araña realiza una maniobra discursiva que permite sospechar, y corroborar a la luz de los manuscritos, que la novela pensada para referir la historia del gobierno de la llamada "Revolución argentina" (representado en los militares Onganía-Levingston-Lanusse) remite, por un cambio de fechas, a referir el gobierno de Isabel Perón que Puig había padecido y que finalmente lo llevan al exilio. Pero mientras que en El beso Puig realiza un "guiño" al lector argentino a partir de un "error" que deja deslizar intencionalmente, ${ }^{8}$ para poder decir lo que el discurso de la censura le hacía callar, en Pubis angelical, habiendo perdido el público argentino, explicita lo que en su novela anterior conformaba su contexto de producción, para llegar a Maldición eterna a quien lea estas páginas con la certeza de una despedida de los tópicos del exilio: el personaje que muere paralizado física y culturalmente. Sin significados, sin referentes, sin lengua, el personaje argentino, el Sr. Ramírez, implica la historia que se necesita olvidar."

\footnotetext{
${ }^{7}$ No incluyo la última novela, Cae una noche tropical (1988), donde el escritor vuelve a escribir en castellano, a cinco años del reestablecimiento de la democracia en Argentina, donde las representaciones del género que allí se ofrecen parecen haberse "estabilizado".

${ }^{8}$ Pese al cambio de fechas del capítulo ocho que se observan en el manuscrito de la novela, permanece el año 1972 para la fecha de detención de Valentín, según detallo luego.

"De Diego explica en su ensayo el concepto de "presentismo" al que se refiere Edward Said en

"Recuerdos del invierno": "La condena al presentismo absoluto imposibilita la aceptación de una
} 
En los apuntes manuscritos previos a la redacción de El beso, una reflexión metatextual no solo nos entera que uno de los comienzos pensados para la novela sería un parlamento en forma de delirio de Molina, el personaje homosexual, sino que los filmes tenían una funcionalidad que iría más allá de la relación de los dos personajes:

"Ese pre-prólogo puede ser una visión extraña de $\mathrm{F}<$ olle $>$ al morir, algo así que da como puntadas ocultas a lo largo de capítulos y film stories, algo en una primera persona misteriosa que no se sabe quién es". ${ }^{10}$

Las “puntadas ocultas", según esa anotación, reiteran los motivos: en todos los filmes está presente el planteo gótico de la identidad distorsionada y la doble naturaleza, la creación de una atmósfera de extrañeza que cuestionan la noción de normalidad; la marca, el secreto, la traición enhebran aquellas "puntadas ocultas"que escanden las claves de la intimidad histórica, un relato desplazado, el enfrentamiento con el orden estatal. Mise en abyme de la historia, los filmes incluidos narran el vampirismo presente en los sistemas autoritarios. Pero esta afirmación sería casi imperceptible si no consideramos su relación con las fichas de presos que habían sido desplazadas del comienzo - otra de las posibilidades contempladas, según los apuntes - hacia la mitad de la novela, además de los testimonios que registrara el escritor de las entrevistas a ex-presos políticos liberados por Héctor Cámpora: los años 1974 y 1975 que figuran en las fichas de los personajes presos Molina y Valentín —en el capítulo ocho- sobreescriben otras fechas que figuraban originalmente, los años 1971 y 1972, en

nueva historia en un nuevo lugar; por momentos, los exiliados dan testimonio de un temor a que el nuevo arraigo se transforme en una actitud complaciente con la dictadura. Las consecuencias del presentismo, de ese tiempo sin historia, es el anclaje en el tiempo y el lugar perdido"(234). De alguna forma - agrego - el Sr. Ramírez es el personaje paralizado por la ausencia (ausencia de memoria) invade con historia su cuerpo. Por otra parte, es significativo el hecho de que Maldición eterna a quien lea estas páginas esté escrita originalmente en inglés, poniendo en el plano autoral, en la escena lingüística de la novela, la despedida de los tópicos, con el desplazamiento de su lengua natal a otras lenguas. En este sentido el multilingüismo se traduce en travestismo de la voz que borra la frontera de lo nacional.

${ }^{10}$ Cat People es la historia que pone en escena toda la novela en miniatura, y que decide significativamente su comienzo. Puig introduce de forma velada el problema de la identidad y la nación a través del planteo de la singularidad y la diferencia. La xenofobia planteada como origen de la leyenda de la mujer pantera en el film de Tourneur (1942) es el fragmento que Molina no cuenta, que dice olvidar. En la película de Tourneur, el relato de la invasión de los mamelucos a Serbia delata una alteración de valores causada por la degeneración de la raza. El pueblo serbio, pervertido, se entrega a la adoración del diablo por maldad; en la película de Molina, el pueblo "de donde es Drácula" se entrega al diablo por hambre. En la película de Tourneur, la animalización de las mujeres, que se convierten en gato si besan a un hombre, refiere una prohibición que se entiende como nacional; el rey John aparece como un emblema del libertador que vence el mal y devuelve el orden anterior, aunque no puede evitar el nacimiento de una raza infame. Una forma de leer, oculta la otra: Molina cuenta el melodrama por el que Irena, la protagonista del film, no puede concretar su matrimonio, y sin embargo ambas lecturas aluden a una marca, un secreto y una traición: una identidad distorsionada, que originó un pasado histórico (la doble naturaleza de origen atávico), el secreto con el que Irena convive mientras la marca no sale a la luz y la traición a los mandatos de la "raza", resistente a la naturalización por las leyendas del Estado. (El género sexual - la "escuela de la explotación”, según Puig- y su enfrentamiento con el Estado). 
la primera versión conservada de la novela." La única fecha que no se enmienda es 1972, la fecha de detención del activista político, que quebraría la verosimilitud de la historia: siendo el año 1974 en la historia de la novela, Valentín aún continúa preso, salteando de este modo la "amnistía" de 1973, que el escritor recordaba especialmente. ${ }^{12}$ Lejos de equivocarse, lo que intenta decir Puig es que el esperado gobierno peronista había retraído sus mecanismos democráticos a los procedimientos que habían sido propios del gobierno militar. $^{13}$

III- 1984 es, entonces, el año en que Puig hace pública, en forma de proclama, su relación con el campo literario y recoloca en el horizonte de discusiones el tema de la escritura en delito. Pronunciada en Holanda, la conferencia, ${ }^{14}$ concentra las preocupaciones que aparecían en muchas de sus declaraciones. Reconocer la tradición cinematográfica y negar la literaria se había convertido en uno de los Leitmotive sobre el cual Puig edifica cierta figura de escritor ${ }^{15}$ caracterizada principalmente por borrar las marcas del padre y de la patria, ambos integrantes de una pareja que había sido nefasta desde los comienzos, pero que, sin embargo, promovió su producción a partir de la negatividad. El "padre" en el campo literario, Borges, había propiciado que toda una generación de escritores se viera indefectiblemente bajo su sombra; la patria, había condenado a Puig a la censura y al exilio, y sin embargo es el lugar hacia donde dice remitir constantemente su escritura: "escribo en el castellano de Argentina y soy leído en traducciones", ${ }^{16}$ protesta en esta suerte de manifiesto que concentra un cuerpo de nociones que se habían reiterado sistemáticamente en diversas épocas. La patria, el territorio nacional, había adquirido nuevamente la forma expulsatoria que había existido tradicionalmente desde su fundación. ${ }^{17}$ Si para un escritor

\footnotetext{
"Se conoce por testimonios de amigos del escritor que hubo otras versiones de la novela que no se conservaron.

${ }^{12}$ Entre los manuscritos se encontraron los apuntes que el escritor tomara a ex-presos políticos liberados por la "primavera"democrática del presidente Héctor Cámpora, que incluso contienen planos de la cárcel, transcripción del lunfardo usado entre los presos, que luego iría a someter a un prolijo proceso de estilización del lenguaje. (Se publicarán en la edición de la UNESCO bajo el título "Apuntes de la cárcel").

${ }^{13}$ Una característica de trabajo del escritor es su minuciosidad en lo que respecta al armado de las historias, pero también en la corrección de las distintas versiones de manuscritos y dactiloscritos, así como de las versiones en galeras en distintos idiomas.

${ }^{14}$ Publicada con el título "Loss of Readership". Utilizaré el original mecanografiado en su versión geneticista, que incluyo como apéndice de la conferencia nunca publicada en su totalidad.

${ }^{15}$ Tomo la noción de "figuras de escritor" de las teorizaciones sistematizadas por M. Teresa Gramuglio en "La construcción de la imagen", donde suma los aportes metodológicos de "estructura de sentimiento" (R. Williams), "ideologema" (F. Jameson) y "campo intelectual" (P. Bourdieu).

${ }^{16}$ El original dice "I've written my first five novels in Argentinean Spanish, but I'm read in translation. The books are in the Argentinean stores but nobody buys them, I have lost my national readership. The silence of the media is maybe the reason why." Remito al apéndice. (Las traducciones incluídas del texto inglés me pertenecen).

${ }^{17}$ La literatura argentina tiene su génesis en una paradoja y en dos rechazos: rechazo de la cultura, tradición y pueblos prehispánicos y rechazo de la lengua española por ser la lengua de los colonizadores. La paradoja: escribir una literatura nacional en lenguas extranjeras prestigiosas. Con ello se demarca una zona de inclusión atada indefectiblemente a la ideología nacionalista. La campaña
} 
la patria es la lengua, en la primera etapa del exilio de Puig - desde el año 73 hasta fines de esa década - es ese "lugar" al que decide pertenecer y dirigir su escritura. Sin embargo también de esa patria se iría a exiliar en la década siguiente cuando el tránsito hacia la residencia del escritor en Río de Janeiro delinea una escritura de traducción que borra las fronteras de lo nacional. ${ }^{18}$ En el mismo artículo, Puig explicita su posición respecto del peronismo, coincidente, con la que aparece de manera desdoblada en Pubis Angelical. La vuelta de Perón, relata, fue conseguida por una alianza de la izquierda con el ala derechista del partido peronista:

la izquierda creyó que el carisma de Perón daría fuerza y cohesión a la izquierda argentina, creían en el dogma nacional y popular, mientras que yo nunca lo creí, para mí él era hábil, pero demasiado egocéntrico y demagogo. Demasiado concentrado en sí mismo como para crear algo más grande que él, tal como un movimiento socialista verdadero.

A partir de The Buenos Aires Affair, figuraba en las listas negras con personas de la izquierda que habían hablado mal de Perón, y después de padecer algunas consecuencias hostiles (el secuestro de su tercera novela y la posterior amenaza telefónica por parte de la Triple A, definida por el escritor como "una organización nazi protegida por Isabel Perón") decide su ida a México. Y afirma

el tiempo me dio la razón: los peronistas habían traicionado la alianza izquierdista tal como yo temía ... < Perón $>$ era hábil, pero demasiado egocéntrico y demagogo. Demasiado concentrado en sí mismo como para crear algo más grande que él, tal como un movimiento socialista verdadero.

Puig lee la historia como un melodrama, y en su relato no faltan los personajes típicos que reaparecen en sus novelas: el Traidor que oscila entre dos lealtades y que solo es fiel al poder (el líder peronista), la Víctima que en la mayoría de las ocasiones aparece privada de su verdadera identidad y condenada a sufrir injusticias (el pueblo), el Justiciero, que en el melodrama salva a la víctima y castiga al Traidor (el guerrillero: en El beso Valentín, militante del ERP; en Pubis Pozzi, definido como socialista pero igualmente encuadrado en las filas de los Montoneros). En Pubis angelical reaparece una subjetividad femenina

al desierto fue una de las formas que inauguraron ese procedimiento expulsatorio que ha fundado una tradición. Remito al estudio de Beatriz Sarlo.

${ }^{18}$ A partir de entonces escribe en inglés, italiano, portugués: la novela Sangre de amor correspondido está escrita sobre la traducción de unas entrevistas con un albañil brasilero (se conservan cerca de trescientas páginas de la transcripción de esas grabaciones), el guión cinematográfico Siete pecados tropicales está escrito y publicado en italiano, Tango Musik y Vivaldi, son guiones inéditos escritos originalmente en inglés, en el caso del segundo existe otra versión en italiano. Las obras de teatro que tenían su versión castellana, como Triste golondrina macho y El misterio del ramo de rosas fueron publicadas, en vida del autor, en italiano. Pero, como afirma Christopher Larkosh, "El exilio de Puig, no obstante, encuentra una nueva índole en espacios intermedios de lenguaje entre los campos establecidos de idiomas nacionales..." "Su estilo multilingüe, pues, no es solo literario, sino también una especie de exilio de los espacios normativos de lo político/sexual que se llama la nación". 
contrapuesta con la de un militante de izquierda que se une a la agrupación de Montoneros para cambiar el Movimiento "porque siempre afirmaron la preeminencia de lo nacional, y por lo menos en sus fases iniciales mantuvieron la concepción interclasista del peronismo" $(122-23)$.

Pozzi se define como

un abogado que se la pasa defendiendo presos políticos, protestando por torturados, buscando desaparecidos con habeas corpus inútiles y presentando escritos más inútiles aún, para que se descubra el nombre de los asesinos de la Triple A, que en realidad son empleados del gobierno.

-El gobierno que vos votaste, responde Ana.

Y Pozzi:-Mea culpa. Pero hay que cambiar las cosas. En el 73 había que optar, la derrota de la dictadura militar se sintetizaba en el voto al peronismo, más allá de la personalidad de Perón. Nosotros no buscamos la violencia, nos encontramos sumergidos en la violencia (124).

Ana también da su visión:

-Para mí este gobierno es el peronismo verdadero, de matones y nazis.[...] Pozzi, vos te imaginaste el peronismo a tu antojo, y te casaste sin conocerlo. Y es hora que la fiera te muestra los dientes (148).

Cuando Pozzi está a punto de volverse a Argentina, Ana le pide que no se vaya:

-Sí, no te quiero ofender, vos tenés muchas cosas buenas, que te las respeto, de veras, pero esa cosa del peronismo [...] Si te quedás acá a lo mejor se te pasa [...] (218).

Lejos de componer una parodia, lo que construye Puig es una forma ideológica que no se identifica con los modos previstos y previsibles de relacionar política y ficción. Su modo particular de trazar esa relación tiene que ver con un modo narrativo que entabla un discurso oblicuo con lo real debido a nuevas condiciones de existencia y de lucha, al mismo tiempo que presenta un modo contrahegemónico al discurso del poder: si bien se había utilizado la identificación para inculcar el sentimiento de adhesión de las clases marginadas y trabajadoras, ${ }^{19}$ leer y contar la historia como un melodrama es representar el imaginario de la imposibilidad, el sentimentalismo de los excluidos y junto a él una visión crítica de su reproducción.

IV- EL AMOR Y LA IGUALDAD

Es el año 1973 y la primavera democrática se vivía con la efervescencia que ansiaban esos años. La vuelta del peronismo de alguna manera representaba la única posibilidad de

\footnotetext{
"Ya en La traición de Rita Hayworth en el "Diario de Esther" aparece el discurso de la niña peronista que se identifica con su líder, Evita. Pueden consultarse los discursos de Eva Perón en los documentos incluidos por Milceades Peña, 1973.
} 
depositar y centrar las expectativas de revolución popular: los derechos de los trabajadores, el voto femenino, entre otros, habían sido indudablemente hechos que habían concretizado una suerte de reivindicación popular, habían ampliado el límite de la participación política, y conciliado diferentes tendencias aparentemente irreconciliables, cada una de las cuales pretendía entender el "verdadero peronismo". ${ }^{20}$ Cuando Perón asume luego de la segunda elección de septiembre de 1973, la agrupación era un crisol de tendencias de izquierda y de derecha. El "peronismo de izquierda", tal como se denominaba a la agrupación Montoneros, comienza a explicarse el entorno, se habla de un grupo de consejeros de derecha que eventualmente tenían desinformado a Perón, que lo mantenían"cercado" y que dictaba la política gubernamental. La ruptura del movimiento con su Líder se produce con el retiro de las columnas de la Juventud Peronista cuando Perón los echa de la Plaza de Mayo, donde se realizaba el acto del día de los trabajadores. La disidencia que presentaron en forma de consigna política era la causa o la explicitación de una situación que se advertía en escena, en cierto modo un pedido de rendición de cuentas por esa presencia derechista que conformaría la Triple A. ${ }^{21}$ En este marco político es que debe encuadrarse la lucha del Frente de Liberación Homosexual en Argentina, gestado en 1971. Sin embargo, a diferencia del Gay Liberation Front, no se trata de una respuesta que se politiza frente a un episodio específico, sino que se inserta en el estado de revolución que le dieron un sentido político más amplio, de crítica social generalizada más que exclusivamente reivindicativa(Perlongher 77-78). ${ }^{22}$ El Frente consideró también que era necesario apoyar al peronismo, que había

20 Sin duda esta síntesis no carece de esquematismos. Habría que aclarar que el ERP (Ejército Revolucionario del Pueblo), un grupo guevarista que comienza a actual con métodos foquistas después de 1969, y cuyo líder Mario Santucho consideró como "error" la caracterizaciôn que habían hecho de Perón y del peronismo: "nos ubicamos en la vereda de enfrente, porque no confiábamos en Perón, como líder revolucionario; por el contrario, considerábamos que él era el único político que podía impedir el triunfo de la revolución. Por otra parte desconocíamos el papel clave del peronismo en la identidad política del país". Roberto Perdía, que había sido secretario político de Montoneros, declara que "la realidad es que la de Ezeiza fue una gran movilización popular programada para el definitivo retorno de Juan Perón, pero todo quedó reducido a un enfrentamiento armado entre sectores del peronismo, que incluían a los que ya trabajaban bajo el ala de López Rega, y terminarían conformando la Alianza Anticomunista Argentina, o Triple A" ("ERP y Montoneros. El gatillo de la memoria", entrevista realizada por Matilde Sánchez, Clarín, 13 de octubre de 1986).

${ }^{21}$ Montoneros fue la tercera organización armada que había tenido el peronismo. Si bien su origen fue un grupo nacionalista y católico, hacia 1969 , sus posiciones evolucionaron hacia un acercamiento al marxismo por John Williams Cooke y Gustavo Rearte. El distanciamiento sobreviene luego de la matanza de Ezeiza, con la llegada de Perón al país, en 1973, y el acto del 1 de Mayo en la Plaza en 1974, cuando la consigna: "Qué pasa, qué pasa General, está lleno de gorilas el gobierno popular" recibió como respuesta el desalojo de la plaza cuando Perón los trata de "estúpidos e imberbes" y la hipótesis del cerco, ante la evidencia de la actitud de Perón, no puede sostenerse más.

22 "Cada conflicto mínimo - desde un docente autoritario hasta los problemas de los villeros o de los productores algodoneros - parecía ser expresión de la única y gran contradicción de la sociedad: la dependencia o la liberación" (José Luis Romero 4). "Tanto la sincera necesidad de liberarse de un machismo profundamente anclado en la sociedad argentina, como la convicción de que esa liberación no podía sino producirse en el marco de una transformación revolucionaria de las estructuras sociales vigentes, constituyen elementos constitutivos del movimiento gay argentino, que aparecen constantemente a lo largo de toda su historia" (Perlongher 78). 
invadido el imaginario popular con la significación de un proceso de liberación como forma de luchar contra la represión y el capitalismo. En las movilizaciones que se realizaron por la asunción del gobierno peronista, en mayo de 1973, un centenar de homosexuales se hicieron presentes en la plaza con un cartel que reproducía un verso de la marcha peronista: "para que reine en el pueblo el amor y la igualdad". En ese verso los homosexuales leían un sentido de liberación no solo política sino también sexual, con lo cual adelantaban las bases teóricas de su manifiesto, "Sexo y Revolución"; 23 pero los peronistas no aceptaron la apropiación del verso. La muestra fue la ruptura que sucedió al debate simbolizado en las consignas, sumado a las actitudes que los mismos militantes de izquierda tuvieron con el Frente. ${ }^{24}$

V- "SER O NO SER", EL ERROR GAY

No queremos que nos persigan, ni que nos prendan, ni que nos discriminen, ni que nos maten, ni que nos curen, ni que nos analicen, ni que nos expliquen, ni que nos toleren, ni que nos comprendan: lo que queremos es que nos deseen (Néstor Perlongher 29).

"No somos putos no somos faloperos [drogadictos], somos soldados de FAR [Fuerzas Armadas Revolucionarias] y Montoneros" era una de las consignas que se escuchaban en las movilizaciones de Plaza de Mayo, y a la que la revista "Somos" respondió desde el título. Por otra parte, la revista "Homosexuales", anterior a "Somos", había publicado en su único número un artículo cuya tesis central afirmaba la idea de que el afeminamiento gay era la contracara del machismo, la reafirmación de la opresión. "Ser" homosexual o "no ser" un homosexual declarado, mostrarse para hacer efectiva una política de esa visibilidad o simplemente serlo sin necesidad de manifiestos, son los ejes en los que se sostienen las discusiones. De modo que una polémica con la izquierda, se enlazaba con la polémica en el interior del grupo y complejizaba el entramado de debates.

En 1990 se publica "El error gay". Como se ve claramente en el final del manuscrito de "Loss of Readership", (remito al apéndice), la matriz del artículo del 90 estaba allí, en ese artículo-manifiesto que historiza su affair con la censura. Cuando se queja, hacia el final, de la actitud de dos críticos gay, en Brasil, por no dar a conocer una "estampa oficial" sobre su modo de vida sexual, Puig especifica lo que en aquella época de debates había sido su postura: "La homosexualidad no existe. Es una proyección de la mente reaccionaria". ${ }^{25} \mathrm{El}$

\footnotetext{
${ }^{23}$ Ha Kang y Emilio Bernini citan en un epigrafe de su trabajo uno de los bastiones centrales del manifiesto del 73: "El sexo mismo es una cuestión política".

${ }^{24}$ Sebreli refiere que el ERP (Ejército Revolucionario del Pueblo) había declarado "con horror que sus militantes eran recluídos en las mismas celdas con los homosexuales", Perlongher relata que las agrupaciones izquierdistas se corrían de lugar en la columna de manifestaciones populares para no quedar cerca de los gays; "finalmente algunos trotskistas y anarquistas aceptaron la contigüidad"(81). ${ }^{25}$ La revista "Homosexuales", según refiere Perlongher, contuvo un artículo que también fue piedra de toque de las discusiones: "Machismo y opresión sexual", donde se afirmaba que el afeminamiento gay era la contracara del machismo (81).
} 
centro de su argumentación confirma la hipótesis: la sexualidad es una actividad banal, y como actividad de la vida vegetativa es ajena a la esfera de la moral, consecuentemente no debería definir identidades. La defensa de las minorías, por tanto, conlleva la posibilidad de la formación de Ghettos que dificultarían la mezcla y la integración, y acentuarían la identificación a favor de la autodiscriminación.

Desarrolla en "El error gay":

Parece que el gran malentendido empezó hace ya muchos siglos por obra de un patriarca que habría inventado el concepto de pecado sexual, con el fin, entre otras cosas, de controlar a las mujeres [...] desde que aquel hipotético patriarca creó el concepto del pecado sexual, del sexo como manifestación demoníaca (cuando no neutralizada por ciertos ritos de brujería) se pasó a dar inevitablemente importancia al sexo. Trascendencia, significados ocultos, peso moral: he aquí el malentendido peligroso, porque incluso los menos reaccionarios, al negar el componente demoníaco de la sexualidad entraban en la dialéctica de los grandes significados y terminaban olvidando la característica más determinante del sexo, que es precisamente su no pertenencia a la esfera moral (32).

En medio de aquella controversia iban tejiéndose los hilos de las versiones de la que sería la cuarta novela de Puig: El beso de la mujer araña responde a esos debates. Ha Kang y Emilio Bernini (70-74) afirman que Puig transcribió en esa novela una disputa consciente de las teorías sobre homoerotismo en las notas al pie de página que explican las conductas sociales de sus personajes. Daniel Balderston (271-277) se ocupó de analizar detalladamente esas teorías, y descubrió debajo del acopio de resúmenes y autoridades que representan las ocho notas al pie, la cita de veintiséis autores y treinta y un textos que en realidad - con tres excepciones - ya estaban citados en las dos fuentes más utilizadas, Homosexuality de D. J. West, 1967, y Homosexual Opression and Liberation, de Dennis Altman, 1971. Y que además, las notas uno a cuatro remiten al primer libro, mientras que de la cinco a la ocho al libro de Altman, que a su vez critica al primero. Puig, afirma Balderston, incluye simplificaciones de títulos, tergiversaciones de opiniones colapso de dos o tres autoridades en uno, o parafrasea material. Todo hace pensar, concluyo, que lo que arma Puig con el famoso "Tratado" de las notas al pie, es un entramado de definiciones que por estilización e hiperbolización llegan a la parodia, donde no faltan las explicaciones científicas que desde el siglo pasado tranquilizaban las conciencias con palabras que nominaban y cercaban a "los raros". El apócrifo (la Dra. Taube, la última nota al pie, en el capítulo once) ni la simplificación, ni la clasificación absurda, las explicaciones que remiten a autoridades que se critican y que en oportunidades se invalidan por contradicción. ${ }^{26}$

La tesis de la nota final de la novela responde desde el título "Sexualidad y revolución" a aquel manifiesto político que había editado el Frente de Liberación Homosexual, "Sexo

\footnotetext{
${ }^{26}$ Lo curioso del uso de estas dos fuentes es que el libro de West es duramente condenado por Altman por su uso del lenguaje de las ciencias sociales de los sesenta: West utiliza sin aparente ironía términos descriptivos que implican un fuerte juicio moral: "normal", "perverso", "desviado". Altman comenta: "however able a psychologist West may be, he is a poor logician, and a man too apt to confuse social prejudice with natural laws" [...] "his use of words like 'natural', 'perversion', etc. powerfully reinforce conventional morality. [...] Like too many psychologists, West is strongly conformist, even where his own expert knowledge tells him that social norms are not necessarily
} 
y Revolución", donde se analizaba el papel de la opresión sexual en el mantenimiento de la explotación y terminaba definiendo el movimiento como "movimiento anticapitalista, antiimperialista y antiautoritario". ${ }^{27}$ Allí se afirma que ninguna revolución es completa ni exitosa "si no subvierte la estructura ideológica íntimamente internalizada por los miembros de la sociedad de dominación". Sin embargo también se critica allí desde la teoría de los "errores" el proceso revolucionario rioplatense (Ha Kang-Bernini). Paralelamente, para la mentada Dra. Taube, la liberación sexual y la liberación gay en particular, son partes esenciales del anhelado cambio social. No puede negarse la filiación marcuseana y leninista de su pensamiento, y sin embargo también está presente, en esta voz travestida, la crítica a la fijación de los roles, otro "error gay". Si la novela es también, por un lado, un ajuste de cuentas con algunos grupos de izquierda, en estaúltima nota al pie la crítica se dirige también al "closet" que puede surgir de la imitación, por parte de los homosexuales, del tradicional modelo burgués e, indirectamente, al "closet" que puede surgir de la defensa ciega de las minorías:

Anneli Taube interpreta así la actitud imitativa practicada hasta hace poco por los homosexuales en alto porcentaje, actitud imitativa ante todo de los defectos de la heterosexualidad. Era característica de los homosexuales varones el espíritu sumiso, conservador, amante a todo coste de la paz, sobre todo a coste de la perpetuación de su propia marginación, mientras que era característica de las mujeres homosexuales su espíritu anárquico, violentamente disconforme, aunque básicamente desorganizado. Pero ambas actitudes resultaban no deliberadas, sino compulsivas, impuestas por un lento lavado cerebral en el que intervenían los modelos de conducta heterosexual burgueses, durante infancia y adolescencia, y posteriormente, al asumir la homosexualidad, los modelos "burgueses" de homosexualidad.

Este prejuicio, u observación justa, sobre los homosexuales, hizo que se los marginara en movimientos de liberación de clases y en general en toda acción política. Es notorio la desconfianza de los países socialistas por los homosexuales. Mucho de esto afortunadamente, acota la doctora Taube-, empezó a cambiar en la década de los sesenta, con la irrupción del movimiento de liberación femenina, ya que el consiguiente enjuiciamiento de los roles "hombre fuerte" y "mujer débil" desprestigió ante los ojos de los marginados sexuales esos modelos tan inalcanzables como tenazmente imitados.

La posterior formación de frentes de liberación homosexual sería una prueba de ello (211).

sensible'[...]" Aparte de las pequeñas supresiones en la traducción de Puig (y de la aclaración que el deseo "normal" femenino se dirige hacia el hombre, que no está en el original), lo interesante de este ejemplo es que West se refiere en su nota no solo a G. L. Foss, "The influence of androgens on sexuality in women", sino también a dos otras fuentes: R. B. Greenblatt, "Hormonal factors in libido", y W. H. Masters y D. T. Magallon, “Androgen administration in the post-menopausal woman”. Es decir que Puig simplifica el aparato crítico, refiriéndose a solo uno de los tres artículos.

${ }^{27}$ Las citas que se incluyen continúan en la nota de la Dra. Taube, el personaje que escribe a pie de página en la novela de Puig: "Marcuse señala que la función social del homosexual es análoga a la del filósofo crítico, ya que su sola presencia resulta un señalador constante de la parte reprimida de la sociedad". Esto proviene, señala Balderston, de Altman, quien resume a Paul A. Robinson, autor de un libro The Freudian Left, sobre Reich, Roheim y Marcuse (276). 
En "El error gay" afirmaba:

yo admiro la obra de los grupos de liberación gay, pero veo en ellos el peligro de adoptar, de reivindicar la identidad "homosexual" como un hecho natural, cuando en cambio no es otra cosa que un producto histórico-cultural, tan represivo como la condición heterosexual (32).

Frente a la seducción de Molina, que igualmente cuenta la historia argentina desde la filmografía de clase B (Julia Romero 145-163) la obsesiva manía explicativa de Valentín, la ratio militante, parece reproducirse en el contrapunto que compone la historia "de arriba", como lo denomina Lucille Kerr, y el texto "de abajo", en las teorías sobre homosexualidad que explican hasta el cansancio y el absurdo el pretendido cientificismo tranquilizante, el denominador que etiqueta y, como en el fin de siglo XIX y comienzos del XX, acorrala la diferencia. $^{28}$

\section{VI- Cuba Y LA REVOLUCIÓN}

"Cuba was discussed, I said I admired the experiment but disliked the mistakes such as the concentration camps for non castrist intellectuals and gay people, and above all, the imitation of the soviet model in some aspects of the civil liberties". Las palabras encerradas en la enmienda del manuscrito relatan, junto con la historia de censuras de la ciue Puig fue objeto, solo un episodio de una historia de progresivo "endurecimiento o stalinización" de la Revolución Cubana, ${ }^{29}$ de la que también hablan Perlongher y Néstor Almendros. UMAPs (Unidades Militares de Ayuda a la Producción) era el eufemismo que daba nombre a esos campos de concentración que funcionaron desde 1964 hasta 1967, donde se recluían homosexuales y religiosos, entre otros marginales para el sistema.$^{30}$ El campo intelectual también mostraba su lado siniestro, ya que el argumento socialista se había convertido en el pretexto según el cual la autoridad de turno se imponía. ${ }^{31}$ Es este el contexto al que se

\footnotetext{
${ }^{28}$ Es interesante contraponer este gesto de la escritura de Puig con el siglo XIX y el rol de los higienistas al desarrollo de una sociedad disciplinaria que buscaba una identidad, - donde esa cuestión sirvió para reivindicar una identidad cultural latinoamericana- aplicar en forma creciente los sistemas de vigilancia hacia "las temibles epidemias de anarquismo, feminismo y homosexualidad" (Salessi 35051). En el momento de la modernización y la reafirmación nacional, las instituciones del Estado estaban compelidas a la formación de ciudadanos identificados como heterosexuales. Para la relación de sujeto y nación en la formación de identidades en la Argentina remito al minucioso estudio de Francine Masiello como al de Josefina Ludmer.

${ }^{29}$ En ese proceso de endurecimiento el "caso Padilla" es uno de los episodios que explicitan el agravamiento de la situación.

30 Ernesto Cardenal testimonia esos horrores en su libro En Cuba (Perlongher 119-125). En 1971 se declara la homosexualidad como "patología social" en el Primer Congreso Nacional de Educación y Cultura.

31 "Al igual que Shumyatsky, el tristemente famoso ministro de cinematografía de Stalin, Guevara Valdés imponía su voluntad absoluta. Terminé por darme cuenta de que estaba trabajando no para el pueblo, como se pretendía, sino para un monopolio estatal, y que la autoridad de turno actúa como cualquier otro productor capitalista e impone sus caprichos de la misma manera y aún peor, solo que recurriendo a pretextos falsamente sociales" (Almendros 47-48).
} 
refiere Puig, y al que alude con carácter alegórico en su cuarta novela. Si en el capítulo ocho, como dijimos más arriba, advertíamos un guiño que refería el autoritarismo de un gobierno democrático, en el capítulo siguiente, ya en la segunda parte de El beso de la mujer araña, se refiere Puig solapadamente a la situación cubana. Los cambios se vuelven significativos a la luz del film de Tourneur, ${ }^{32}$ al que traiciona notablemente: la isla se presenta como el "paraíso" de la explotación, donde los trabajadores son convertidos en zombis en medio de una plantación de bananales. El film relatado por Molina ubicaba los hechos, en su primera versión, en medio de una plantación de cañaverales, que luego reemplazó por bananales, excepto en una sola oportunidad, que se conserva en la versión édita:

Y ahí hay un detalle que te pone en la pista de que algo le pasa, porque él lo primero que hace es presentarle a la chica al mayordomo, que es un cincuentón, pero francés, y el mayordomo le pide que el muchacho le firme ya en ese momento unos papeles, del embarque de bananas en ese mismo barco que la trajo a la chica, y el muchacho le pide que más tarde, pero el mayordomo, le insiste, y el muchacho lo mira con odio y cuando va a firmar los papeles se ve que no tiene casi pulso para escribir, le tiembla la mano. Y todavía es de día, y toda la comitiva que llegó en los carritos con flores está en el jardín esperando a la pareja para brindar, $y$ traen de todos jugos de frutas, y por ahí se ve a unos delegados de los peones negros de los cañaverales que traen un barrilito de ron como obsequio al patrón, pero el mayordomo los ve y se pone furioso, y con un hacha que hay ahí cerca le da unos hachazos al barril y todo el ron se cae al suelo (164-165).

El relato subraya el modo semioculto con que Puig desea hacer notar el suceso. Lo que parecía un error del escritor, imposible por otra parte, por el modo meticuloso de trabajo, ${ }^{33}$ recuerda la Cuba castrista, cuando miembros del Gay Liberation estadounidense se unieron en la brigada de cooperación internacional "Venceremos" para ir a cortar caña a la isla. Como muestra de resistencia a la opresión stalinista, el gesto hacía coincidir, al igual que la Dra. Taube, revolución erótica y revolución social. ${ }^{34}$ Puig esquiva el sentido único, que considera un "error". Si utiliza el discurso médico, lo coloca en otro lugar, lo anula como lugar de legitimación. Sus desplazamientos vinculan y desvinculan, nominan y desestigmatizan, en un tránsito de cuerpos de relatos y de cuerpos que relatan, que barren las fronteras poniendo "en juego" la vida en la misma fuga y el deseo en la liberación.

A diferencia de la política de la visibilidad de "los raros" de fin de siglo, Puig escribe una poética que responde y prefiere la sutileza de los guiños en una narrativa de la seducción, donde se elude la identificación y se evoca la libertad, la complicidad, el corazón del gran secreto.

\footnotetext{
${ }^{32}$ Nos referimos a $I$ walked with a zombie, en el cual Puig dijo haberse inspirado.

${ }^{33}$ Los manuscritos muestran las sucesivas etapas de corrección de los que eran objeto, así como las pruebas de galera de las diferentes versiones, que revisaba personalmente. Teniendo en cuenta, además, que hasta las calles que recorre Molina, en el capítulo quince, durante la persecución de la policía, fueron cotejadas con un mapa de Buenos Aires, según consta en las anotaciones.

${ }^{34} \mathrm{El}$ relato del suceso lo refiere Echavarren (246-47).
} 


\author{
APÉNDICE: \\ LOSS OF A READERSHIP \\ (Transcripción geneticista del manuscrito) \\ Manuel Puig
}

Nota: Para la transcripción del manuscrito he tratado de simplificar los signos que indican las variaciones textuales a dos: el uso de letra negrita, para indicar palabras o frases agregadas; y letra cursiva para indicar palabras o frases tachadas en el texto original.

I've been asked by SLAA to tell you about my long affair with censorship. It is a long story indeed, it started in 1966 with my very first manuscript and quite a complex story one complex. But let me brief you quickly about my background, I was born and raised in Argentina was finishing my philosophy studies when I won a scholarship to study film direction in Rome, back in 1956. That was real vocation, it seemed, so I left everything and sailed to Cinecittà. But By 1962 I had found out the movies were not my habitat, as an assistant director I was hating team work, mainly because of my difficulty to impersonate authority or to deal with it; and as a script writer I was finding that out that the themes I wanted to develop demanded more space that the average 90 minutes of a film. One of those film scripts outgrew its screen mold and became a novel. It happened simply that a three lines monologue by one of the secondary characters became a bit long for film standards: I listened to the character talk and it went on for thirty pages. That was the first chapter of $m y$ a first novel, something I had never planned on doing. Immediately I felt that it was a safer territory for me, I could do it my work alone and with the possibility of redoing it over and over again. I abandoned Rome and the film industry and went to New York, looking for any job that would allow me free hours for completing my novel. It took me three years and $a$ half to do it, and then other three years to see it published. The title was "La traición de Rita Hayworth", later translated into Dutch by Meulenhoff as Het verraad van Rita Hayworth. But that was much later. In 1965 I first showed the manuscript to a friend of the school film school in Rome, Néstor Almendros, later to become an Oscarwinner for his photography work. I didn't know how to handle the situation. Néstor showed the manuscript to the already established Spanish writer Juan Goytisolo, who found my work interesting and presented it to his publisher in Barcelona. Dictator Franco was still alive and the censorship system in Spain had an office where publishers could submit their problematic manuscripts for approval before venturing into a publication. My novel presented a problem: it just dealt with children and housewives in a small pampas town, it had no explicit political ideology, but its implicit criticism of the system was unpleasant to the censors. Worse than that, some of those children spoke the type of foul language that usually hides a terrific fear of the forthcoming sexual initiation, and that really irked the censors. Anyway there was no rejection of the total manuscript, some kind of negotiation was asked for. I was working then as an airline clerk at Kennedy Airport and used a free ticket to fly to Barcelona. The meeting with the publisher was a disaster, first of all our personal styles clashed, he was dressed to the teeth, I was merely covered. He was rich and a bon vivant and a Communist; I was poor, of frugal habits and only a socialist; But I must admit I was not too tactful with the 
Barcelona gentleman him, I accused some intellectuals of doing free tourism is Cuba and giving Castro adulation instead of constructive criticism. He was just back from Cuba, a fact I didn't know. Cuba was discussed, I said I admired the experiment but disliked the mistakes such as the concentration camps for non castrist intellectuals and gay people, and above all, the imitation of the soviet model in some aspects of the civil liberties. I immediately fell off grace with the Barcelona gentleman and the book was not published, one year later the reason given was "censorship prohibition", which was not true. So my first encounter with censorship was a strangely mixed affair, as far as ideology factors were concerned. Dictator Franco was not entirely to be blamed. But strange as it could sound, it was fairly indicative of the things to come.

In 1967 I left New York and landed in my native Argentina after eleven years abroad. The literary director of an important publishing house wanted to publish the novel but Argentina in those days was under a military regime, the one by General Onganía, 19661970 , and the censorship conditions were quite peculiar. There was not a central office as in Franco's Spain, apparently publishers were free to sell anything but if some government official in the Mayor's office or wherever, objected to the book, it could be seized for subversion against the authorities or for pornography or for whatever, and then four people were incarcerated: the author, the publisher, the printer and the owner of the book store where the book had been sold. Well, that made a publisher quite apprehensive, and for a good reason. Anyhow, that literary director I told you about was very brave, his name is Paco Porrúa, and he skipped the surveillance of other people in the house and sent the book to the printer's. The first galleys came without trouble, but in the first page-proof a linotypist noticed the abundance of four-letter words and stopped the work. He took the material to a superior and that was the end of the edition. Evidently the linotypist didn't want his boss to go to jail. Now, the peculiar point in this case is that linotypists in Argentina in those days read word by word, not phrases, and that was what made him panic. If he had read phrases he would have noticed that it was just frightfully inexperienced and innocent twelve to fourteen year old boys talking and trying to live up to the machismo of the elder. Pathetically childish, not pornographic.

A year later another Argentinean publisher, well known for not paying anybody royalties, wanted to go ahead with my half-printed novel. The political tension was even bigger than a year before and everybody expected the book to be seized by the police. Finally, in 1968 the book was published, to indifferent reviews, it sold very little and nobody noticed it's appearance. In the meantime Juan Goytisolo had convinced Gallimard to publish La traición de Rita Hayworth and in 1969 it appeared in french and was selected by Le Monde Among the five best foreign books of the year. My second novel was published that same year in Argentina, it was Boquitas pintadas, later published in dutch by Agathon as De latste Tango. Suddenly the Argentinean readers discovered me and I became a bestseller. At the same time the Onganía regime was dying so there were no censorship attacks. I became quite popular in my country, and the honeymoon lasted until 1973 when I published my third novel, "The Buenos Aires affair".

However, even in 1969 there were shadows gathering in the horizon. Cubahad decided not to print my books, it seemed they were considered too concerned with sexuality, and worse of all, with homosexuality. 
1973 was the year of the return of Perón to Argentina, brought back by an alliance of the Left with the old peronist party, a mostly right wing one. I didn't like this merge, my friends in the Left that were joining that stream believed firmly that Perón's charisma would give strength and cohesion to the Argentinean Left, and they believed also that Perón's populist but vague dogma was deep down a socialist one. I never thought so to me he was skillful egoist demagogo but too self centered to create something bigger than himself. When he was ousted from government in 1955 he chose Franco's Spain for a residence, and he was in the best of rapports with the Spanish dictator. That was one of the many evidences of his real political tendencies. As history tells, the moment he came back into power he eliminated the leftist members in his cabinet.

Now let's go back to that third novel, The Buenos Aires Affair. I have never believed in explicit political novels, I've never written one. I want to deal with real characters, and if they are so they inevitably establish a political environment, even if untold. The main male character in my novel had been a college student during Perón's first term, 46-52, he had then been in a leftist student movement and that had meant harassment by the peronist police. I couldn't eliminate that from the novel, it would have been unfair to the character, to history, to the truth I try to find out by means of my novels. My left-wing friends thought that was most inopportune, in 1973 Perón was the choice of the Left and criticizing him was a reactionary piece of nonsense. That was their point of view. I kept sticking to mine. I believe in a democratic critical attitude, that doesn't mean sending water to the enemy's mill, as some people think. In my novel there was mention also of Perón's good points, the creation of labor laws, for instance. But in 1973 there was room only for praise for him, criticism was blasphemous.

A few weeks after publication I started to feel a certain hostility in the media and left the country for a couple of months. I thought he was far too shrewd to launch a witch hunt, but then one day the first act of literary censorship took place, my book was chosen for this honor. It was seized for pornography reasons, and a few book store owners spent one symbolic night in jail because of me. I never went back to Argentina, it will soon be twelve years of that. I think I was right about Perón reluctance to a wild witch hunt but he died before one year in government and then his wife did start the wildest witch hunt the country has memory of, continued with relish by the Military Junta that replaced her in 1976.

In December of 1974 it had been more than a year I had left Argentina, but anyhow the Triple A, a nazi organization protected by Isabel Perón, called my parents house to ask me to leave the country in 24 hours... or else. I was in a long list with left-wing people who had endorsed peronism.

I lived for a while in Mexico, then in New York by end of 1976 I had published my following novel in post Franco Spain, "El beso de la mujer araña", published later in dutch by Agathon as "De Kus van de spinnevrouw" No argentinian publisher had dared do it my novels had been translated into many languages and that allowed me to live in exile without problems, and without luxuries either. The eastern European countries only published "Boquitas pintadas", the rest was considered too concerned with eroticism. When the Military Junta took over Argentina in March 76 I thought they would allow "The Buenos Aires Affair" back in book stores, since it was considered an antiperonist novel, but its prohibition was renewed and emphasized. In October 76 there was a Latin-American 
celebration at the Book Fair in Frankfurt and I was invited. I knew that there I was going to see some of those Left-wing intellectuals who had objected to the antiperonist overtones of "The Buenos Aires Affair" I thought they had already reconsidered their ideas, time had proved me right, peronists had betrayed their leftist allies just as I had feared. Well, I arrived in Frankfurt and I felt immediately a funny atmosphere, history hadn't taken place, nobody had acknowledged reality, Perón hadn't kicked the leftists in their pants, I was still a reactionary for not having joined the movement. Worst of all my book had been banned by the right wing and the Argentinean left didn't care much about it.

By the end of 1976 I had published my following novel in post-Franco Spain, El beso de la mujer araña, published later in Dutch by Agathon as De kus van de spinnevrouw. No Argentinean publisher had dared do it. This novel presents a homosexual and a political activist in a Buenos Aires prison cell, two people trying to change reality in their respective manner. It was a book to irritate the Junta very much and my parents were still living in Argentina, I was terrified for them. A few months before publication I started to have every night the same nightmare, a bomb exploding in my New York apartment and in my their home. I begged the Spanish publisher to halt it's publication, they did, but after six months they were having a financial problem with the stored edition and they let it loose. Well, with all the story of the banning of my books by the clearly extreme right wing Junta, I thought the international left wing publishers would be quite ready to publish this new book in translation. I was wrong, Feltrinelli, my publisher in Italy, turned it down and so did Gallimard. They didn't like the mixture of sexual liberation with social revolution, something that I wanted to present as different aspects of the same struggle for human dignity. The reviews in Spain were lukewarm, so after so much apprehension and the novel didn't cause any special immediate effect. It was quietly banned in Argentina and other dictatorships in South America. But elsewhere, with the time, it became my better known book, now I'm known as the author of "Kiss of the Spider Woman". It had been translated into many languages and everywhere it has found both attacks and praise, but the good amount of press attention anyhow. In colleges it is constantly analyzed and, has for instance, it has been obligatory reading in the national program of the French University for a couple of years now. A play was derived from it, in my own adaptation, the film version is been shown in Cannes these days and the distinguished German musician Hans Werner Henze is preparing an opera composing an opera based on it. But it all happened quite slowly.

After this novel came other three, and finally in September of 83, when the Junta was leaving Argentina, the Spanish edition of "Kiss of the Spider Woman" arrived at the bookstores in my country. A year and a half has passed by since then and there has been not one review or comment in the now free Argentinean press. Even during the Junta there was some slight mention of my later books, always from lukewarm to negative, but concerning "Kiss of the Spider Woman", not one line. So now I'm published in Japan, Israel, Cuba finally, Sweden, Poland finally, but I'm not of interest to my fellow Argentineans. I've written my first five novels in Argentinean Spanish, but I'm read in translation. The books are in the Argentinean stores but nobody buys them, I have lost my national readership. The silence of the media is maybe the reason why.

At the present moment I'm living in Brazil, that is since 1980. Kiss of the Spider Woman was a big best-seller there, and the play was for three years on the stage. The gay 
groups endorsed Only the militant gay groups didn't endorse the play, they didn't like the fact that I wasn't showing a heroic homosexual, a model for generations to come. I wanted to portray a real gay character, like many I know. There was hostility but the way the audiences came to like to like this character appeased them somehow. By 1982 I had become quite well known in Brazil and the militant gay groups were pressuring me to make declarations about my sexual ways, so to officiate announce my official "coming out of the Closet". I felt something wrong about $i$ and giving an official stamp to my sexuality and preferred to keep my privacy. That produced a small secret war and a new form of censorship was born. In 1982 another play of mine opened in Rio de Janeiro, and the two gay critics reduced the play to ashes. Since they are of a certain influence, their boycott harmed the commercial career of the play. Now I'll try to explain to you what I don't see right about the matter. It's undeniable that the gay liberation groups have achieved arrive in the field of labor laws, etc., but as with the "Black is beautiful" motto, there is the danger of fortifying the walls of the Ghetto and only gain more alienation. I'm all for integration. And in Sexual matters I only see one radical way to clarify the air, I know it may sound utopic but first of all one thing has to be established: sex has no moral weight, it is an activity of the vegetative life, such as eating and sleeping. The differences in sexual taste are as banal as the differences in taste for food or in ways of sleeping. You sleep with a pillow or without a pillow, that is an act of total banality that does not modify your identity. You eat spicy or not, who cares? Sex is an act of total insignificance, all fun and games, as long as the parties

agree consent. Sex shouldn't define anything. But! many centuries ago the concept of sexual sin was invented and that ruined everything. It seems it was the idea of a man, some pervers vicious patriarch that dasn 't having for enough and created the roles of sainted wife and ridden sinful street woman, so to have the exciting contrast between the woman at home and the one outside. But humanity is paying for that vicious idea ever since. The moment you give sex a moral dimension it is adulterated, since it's nature is innocence itself, pure instinct for pleasure. Now, you may say how can sex be consider banal when it is at the very origin of life. Well, I think the very origin of a new human being should be in the desire wish of their parents to see themself create the child, and not in an accident. The moment that sexuality is given significance, moral weight, etc., the sexual rolesare created, magnified, and people are put under pressure at an early age to assume roles a certain sexual behaviour for which they are not always suited, they are asked to define their taste when it is still vague and should stay vague if it was the case. I think sexual roles are in general a product of social pressures and not the product of real human needs. If sex was not considered transcendental and morally significant, it would be taken lightly and the real sexual needs would come into the surface of each one. The result of the actual state of things is one of repression and adulteration of the real sexual drives. That's why I don't think the formation of a "gay" identity is right. Identity shouldn't be defined by a sexual activity, because sexual activities should be considered of no significance. There shouldn't be such a thing as a heterosexual, or a homosexual. Homosexuals don't exist, there are persons who practice homosexual acts, but that banal aspect of their lives shouldn't define their personality establish their identity. Homosexuality does not exist, it is a figment of the reactionary mind. 


\title{
BIBLIOGRAFÍA CITADA
}

\author{
Bibliografia de Manuel Puig
}

Dactiloscrito de "Loss of Readership" (Versión publicada, en forma parcial: "London conference on censorship. Writers and repression"en la revista Index on Censorship. Londres-Estados Unidos 13/5 (5 de octubre de 1984): 28-31. En Argentina el artículo al que nos referimos se publicó parcialmente en Primer Plano, suplemento cultural de Página/12 el 5 de diciembre de 1993, bajo el título "Censuras y rencores (La pérdida de un lectorado)", traducción de José Amícola.

Manuscrito y dactiloscrito de El beso de la mujer araña. Versión publicada: Barcelona: Seix Barral, 1976.

"El error gay", incluido en la revista El porteño, año IX, septiembre de 1990.(El texto original fue escrito en italiano con el título "La omosessualità non existe"): 32-33. Pubis angelical. Barcelona: Seix Barral, 1979.

Maldición eterna a quien lea estas páginas. Barcelona: Seix Barral, 1980.

\section{Biblografía CRÍTICA}

Almendros, Néstor. Días de una cámara. Barcelona: Seix Barral, 1990.

Avellaneda, Andrés. Censura, autoritarismo y cultura: Argentina 1960-1983. Centro Editor de América Larina, 1986.

Balderston, Daniel. "Los progresos de la doctora Anneli Taube". Encuentro Internacional Manuel Puig. José Amícola Graciela Speranza, comp. Rosario: Beatriz Viterbo, 1998. 271-277.

De Diego, José Luis, "Notas sobre exilio y literatura". Encuentro Internacional Manuel Puig. José Amícola Graciela Speranza, comp. Rosario: Beatriz Viterbo, 1998. 227236.

Echavarren, Roberto. "Identidad versus vapor". Encuentro Internacional Manuel Puig. José Amícola Graciela Speranza, comp. Rosario: Beatriz Viterbo, 1998. 245-258. Arte andrógino. Estilo versus moda en un siglo corto. Buenos Aires: Colihue, 1998.

Ha Kang, Jung y Emilio Bernini. "Por qué seremos tan hermosas. Frente de Liberación Homosexual: una política del loqueo". El ojo mocho. Revista de Crítica Cultural 11 (Buenos Aires, Primavera de 1997): 70-74.

Gramuglio, M.T. "La construcción de la imagen”. La escritura argentina. Tizón, Rabanal, Gramuglio. Universidad Nacional del Litoral, 1994.

Kerr, Lucille. "Manuel Puig El beso de la mujer araña". Hispamérica 21 (1978): 71-81. Larkosh, Christopher. "Manuel multilingüe: traducción, tránsito intercultural y entrelugares literarios", ponencia leída en el Encuentro Internacional Manuel Puig. Universidad Nacional de La Plata, 1997.

Ludmer, Josefina. "1880: los sujetos del estado liberal”. La situación autobiográfica. J. Orbe, comp. Buenos Aires: Corregidor, 1995. 69-76.

"Mujeres que matan". Revista Iberoamericana LXII/176-177 (julio-diciembre 1996): 781-797. 
Mallea, Eduardo. Historia de una pasión argentina. Madrid: Espasa Calpe, 1940.

Masiello, Francine. Entre civilización y barbarie. Mujeres, Nación y Cultura literaria en la Argentina moderna. Rosario: Beatriz Viterbo, 1997. (Between Civilization and Barbarism Women, Nation, \& Literary Culture in Modern Argentina, Nebraska, 1992).

Perlongher, Néstor. Prosa plebeya. Ensayos 1980-1992. Selección y prólogo de Christian Ferrer y Osvaldo Baigorria. Buenos Aires: Colihue, 1997.

Peña, Milcíades. Peronismo. Selección de documentos para la historia. Buenos Aires: Ediciones Fichas, 1973.

Romero, Julia. "Puig, la seducción y la historia". Cuadernos Angers-La Plata. Université d'Angers, Francia, y Universidad Nacional de La Plata, Argentina, 1996. 145-165.

Romero, José Luis. "La utopía y el drama". Clarín, segunda sección (13 de octubre de 1986): 2.

Sánchez, Matilde. "ERP y Montoneros. El gatillo de la memoria", entrevista realizada a Julio Santucho y Roberto Perdía. Clarín, segunda sección (13 de octubre de 1986): 17.

Sarlo, Beatriz. "Oralidad y lenguas extranjeras". Orbis Tertius 1. Centro de Teoría y Crítica Literaria, Universidad Nacional de La Plata, 1996. 3-20.

Sebreli, Juan José. Escritos sobre escritos, ciudades bajo ciudades. Buenos Aires: Sudamericana, 1997.

Said, Edward. "Recuerdo del invierno". Punto de Vista 22 (diciembre de 1984): 3-7.

Tizón, Rabanal, Gramuglio. La escritura argentina. Universidad Nacional del Litoral, 1994. 
